\title{
Puces à cellules et génomique fonctionnelle
}

> À l'interface du vivant et de l'inerte, se développe un ensemble de nouvelles technologies regroupées sous le terme générique de biopuces. Grâce à la miniaturisation, nous pouvons imaginer que, demain, de nombreuses études biologiques et médicales se feront avec des biopuces qui permettront d'accroître de plusieurs ordres de grandeur le parallélisme des analyses, les vitesses de réaction des tests et leur débit, tout en réduisant les coûts. Cette évolution a démarré avec l'apparition des puces à ADN et se poursuit aujourd'hui avec, entre autres, les puces à cellules qui permettent d'accélérer considérablement l'étude des gènes de fonctions inconnues et leurs implications potentielles dans différentes maladies. Bien que la technologie en soit encore à ses prémices, il est vraisemblable que les puces à cellules feront évoluer la biologie et la médecine de manière significative. <

\section{Yoann Roupioz, David Castel, Amandine Pitaval, Sandrine Baghdoyan, Xavier Gidrol}

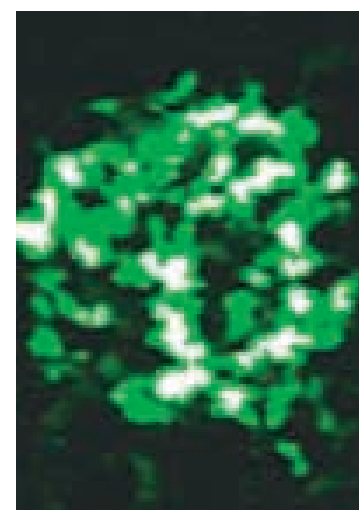

à l'équilibre des $A R N$ messagers (ARNm) d'une cellule. Les bioétudier les ARNm individuellement (Northern blots, RT$P(R .$.$) ), mais, grâce aux puces à ADN, ils purent changer$ d'échelle en analysant simultanément l'ensemble des transcrits d'un génome. De la même manière, pour augmenter la vitesse d'annotation fonctionnelle des génomes, de nouveaux développements technologiques, et notamment de nouvelles biopuces, seront nécessaires.

\section{Transfection massivement parallèle sur puce} étudier les perturbations cellulaires résultant du gain ou de la perte du produit d'un gène. Cette approche a été mise en œuvre avec succès dans des cellules de mammifères ou à l'échelle d'un animal (souris transgénique ou souris knock-out, KO). Toutefois, la plupart des techniques utilisées jusqu'à présent ne permettent l'étude que d'un gène à la fois. Pour répondre aux besoins de la biologie à grande échelle et augmenter la vitesse d'exploration fonctionnelle des génomes, il est nécessaire d'accroître significativement le débit d'analyse.

Au milieu des années 1990, le développement des puces à ADN [1-3] a permis, pour la première fois, d'obtenir une mesure massivement parallèle de la concentration

Article reçu le 29 septembre 2004 et accepté le 9 février 2005
L'analyse à haut débit du gain et de la perte de fonction d'un gène dans des cultures cellulaires passe nécessairement par une étape de transfection massivement parallèle. Quelques publications récentes ont décrit un format de transfection «inverse» et simultanée de plusieurs milliers d'acides nucléiques différents sur puce $[4,5]$. Très souple et d'un coût réduit, ce format dérivé des puces à ADN est en fait assez simple à mettre en œuvre: des plasmides mélangés à un polymère et à un agent de transfection sont déposés sur une lame de verre (lame de microscope) au moyen d'un robot de dépôt muni d'aiguilles creuses, traditionnellement utilisé pour la fabrication des puces à ADN. Chaque dépôt fait environ $200 \mu \mathrm{m}$ de diamètre. La lame est ensuite introduite dans une boîte de culture et recouverte d'une couche uniforme 
de cellules adhérentes. Les plasmides déposés sur la lame vont alors diffuser localement et pénétrer dans les cellules en contact direct avec le dépôt, d'où l'appellation de transfection inverse (Figure 1).

Quelques auteurs ont utilisé des plaques de microtitration pour réaliser des analyses fonctionnelles à l'échelle génomique $[6,7]$. Les puces à cellules transfectées offrent plusieurs avantages par rapport aux plaques de microtitration. En premier lieu, le parallélisme est bien supérieur puisque nous sommes en mesure de déposer 10000 gènes, soit l'équivalent de plus de 100 plaques de microtitration à 96 puits, sur une simple lame de microscope. À terme, l'intégralité du génome humain pourra être analysée sur une seule puce puisque les robots de dépôts les plus récents permettent de réaliser jusqu'à 40000 dépôts sur la surface d'une lame. En second lieu, la miniaturisation des systèmes a l'avantage de diminuer largement les volumes de travail. Cela est particulièrement pertinent pour l'étude de cellules rares, comme les cellules souches, ou pour diminuer les quantités utilisées de réactifs onéreux, comme par exemple les siARN (short interfering RNA) issus de la synthèse chimique. En outre, puisque plusieurs milliers d'îlots de cellules peuvent être analysés sur une seule lame, il n'est pas nécessaire de faire appel à des robots coûteux de manipulation de liquides ou d'analyse à haut débit. II est tout à fait envisageable de déposer différents acides nucléiques au fond de chaque puit d'une plaque de microtitration, pour produire des matrices (les puits de la plaque de microtitration) de matrices (les dépôts au fond d'un puit) d'îlots de cellules. Ce format semble particulièrement intéressant pour le criblage à haut débit de principes actifs ou de molécules pharmacologiques.

\section{Gain ou perte de fonction dans des îlots de cellules transfectées}

Grâce à la transfection massivement parallèle sur puce, des collections entières de gènes humains, clonés dans un vecteur d'expression, peuvent être utilisées pour étudier l'effet du gain de fonction de chacun des gènes, grâce à la synthèse de la protéine correspondante dans des îlots de cellules transfectées. Il est alors possible d'analyser les phénotypes d'intérêt (apoptose, prolifération...) résultant de cet excès de protéine dans les îlots de cellules. Ainsi, notre équipe dispose d'une collection de 16000 ADN complémentaires (ADNc) (dont $70 \%$ sont pleine longueur) clonés sous le contrôle d'un promoteur fort permettant une synthèse exacerbée du produit des gènes. Plu- sieurs équipes dans le monde travaillent activement à la constitution de collections complètes de gènes humains clonés dans des vecteurs d'expression.

La découverte de l'interférence par l'ARN (ARNi) permet l'extinction systématique de l'expression d'un gène (grâce à des siARN) dans des cellules de mammifères, ce qui, jusqu'alors, était particulièrement difficile [8, 9]. Même si l'utilisation de l'ARNi diffère de la technologie knock-out dans la mesure où l'extinction complète de l'expression du gène est difficile, cette caractéristique n'est pas forcément problématique et peut même constituer un avantage majeur pour étudier les protéines dont l'absence totale s'avérerait létale pour la cellule. En outre, l'induction temporelle de l'extinction grâce aux ARNi permet de réaliser des cinétiques d'extinction graduelle du gène étudié et éventuellement d'en observer les effets initiaux. II est donc possible d'utiliser les puces à cellules pour étudier la transfection de cellules par des milliers de siARN en parallèle et analyser les phénotypes d'intérêt résultant de l'absence de la protéine dans les îlots de cellules modifiées [5, 10-12] (Figure 2). Alternativement, il est aussi possible d'utiliser la transfection de cellules par des plasmides permettant la transcription in vivo de l'ARN interférent (short hairpin RNA, shARN). Ces vecteurs d'expression particuliers peuvent supprimer l'expression d'un gène de la même manière qu'un siARN.

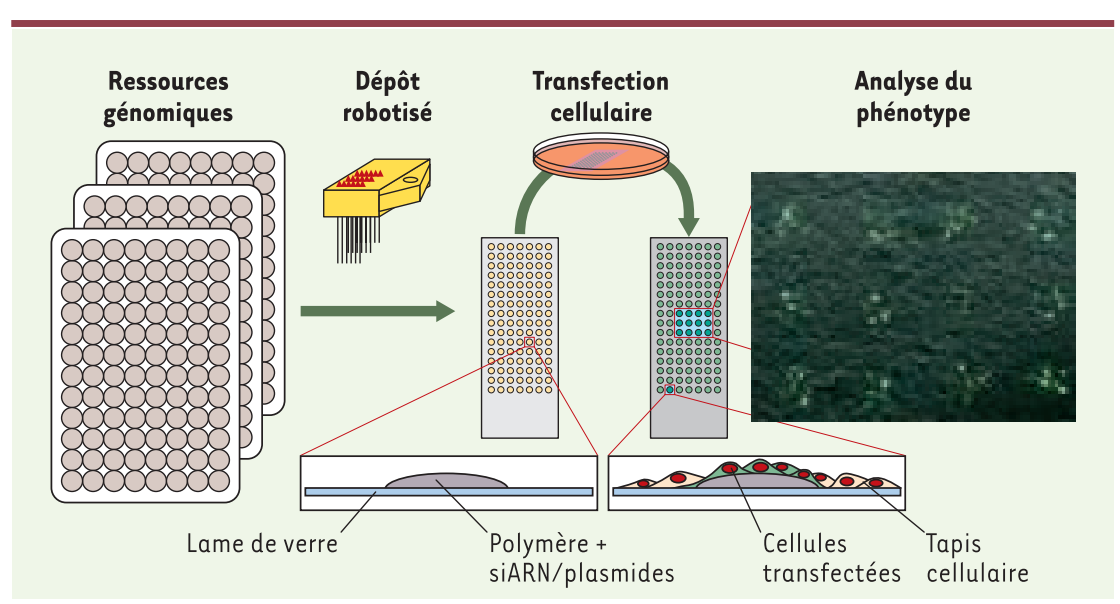

Figure 1. Principe de la puce à cellules pour la génomique fonctionnelle. Un mélange d'acides nucléiques, de polymère et d'agent de transfection est déposé avec un robot de microdépôts sur une lame de verre. Les ressources génomiques utilisées, idéalement des collections pangénomiques, peuvent être de l'ARN (siARN ou shARN, short hairpin RNA), ou de l'ADN (ADNc ou ORF, open reading frame, clonés dans des vecteurs d'expression). Une lame peut ainsi contenir plusieurs milliers de ces dépôts. Les cellules sont ensuite étalées sur les dépôts d'acide nucléique et l'agent de transfection permet la pénétration des acides nucléiques uniquement dans les cellules en contact direct avec le dépôt. Le phénotype des îlots de cellules transfectées peut être ensuite analysé. Les cellules transfectées avec un vecteur d'expression codant pour la GFP (green fluorescent protein) apparaissent en vert, à droite, sur la figure, alors que les cellules non transfectées sont visibles en gris. 


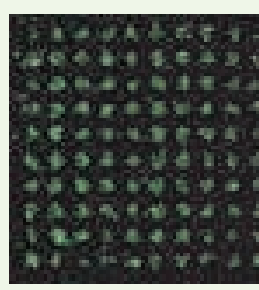

Transfection des cellules par un ARNi spécifique de I'EGFP

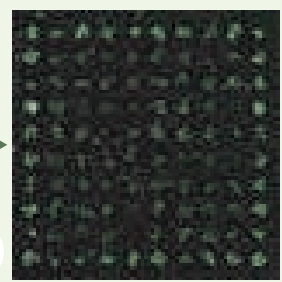

Figure 2. Interférence par l'ARN sur puce à cellules. Les 36 dépôts les plus à l'extérieur dans les deux panneaux $(A, B)$ contiennent un vecteur d'expression codant pour la protéine fluorescente EGFP (enhanced green fluorescent protein) et servent de témoin. Ils entourent un carré de $8 \times 8$ dépôts du même vecteur d'expression contenant également un ARN interférent spécifique de la lamine $A / C(A)$ ou de l'EGFP (B). Après transfection de cellules de rein humain, l'extinction de la fluorescence n'est observée que dans le panneau $\boldsymbol{B}$. Ce résultat montre un défaut d'expression et une perte de fonction de l'EGFP en présence d'un ARN interférent spécifique.
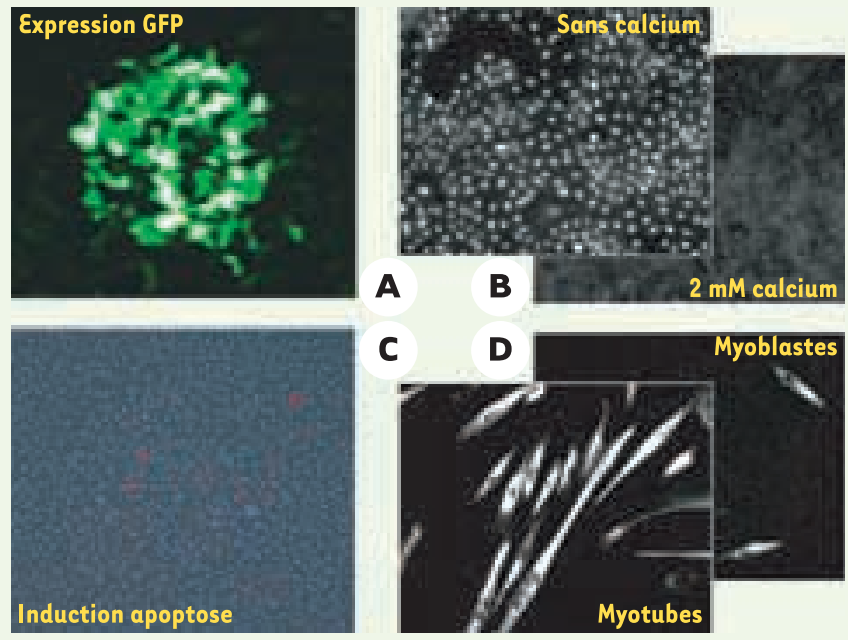

Figure 3. Analyse phénotypique résultant d'un gain ou d'une perte de fonction dans les îlots de cellules transfectées. La détection de cellules fluorescentes après transfection par des vecteurs d'expression codant pour l'EGFP ( $A$ ) a permis la mise au point de logiciels d'analyse des puces à cellules. Ces outils, combinés à des marqueurs spécifiques, devraient permettre, à terme, d’identifier des gènes impliqués dans différentes fonctions cellulaires. À titre d'exemple, nous étudions au laboratoire les gènes impliqués dans la prolifération cellulaire de kératinocytes humains ( $\mathrm{HaCaT}$ ), par immunofluorescence avec un anticorps fluorescent anti-PCNA, en l'absence de calcium (cellules en prolifération) ou en présence de $2 \mathrm{mM}$ de calcium (cellules différenciées) (B). De même, nous pouvons étudier, chez la souris, la différenciation de myoblastes en myotubes par immunofluorescence avec un anticorps anti-myosine qui marque spécifiquement les cellules différenciées (D). Mais d'autres phénotypes peuvent être étudiés, comme l'apoptose, qui est, dans ce dernier exemple, induite par la surexpression de la protéine DR5 dans des cellules humaines HeLa et détectée par l'activité des caspases (C).

\section{Analyse phénotypique}

Les perturbations cellulaires produites dans des cellules cultivées sur ce type de biopuces peuvent être suivies à l'aide de plusieurs approches. Une fois le tapis cellulaire fixé, l'analyse phénotypique peut être réalisée selon différentes techniques: immunofluorescence (IF) à l'aide d'anticorps spécifiques de cibles d'intérêt, hybridation in situ, pour localiser au niveau subcellulaire des ARN messagers, et autoradiographie, pour suivre des biomolécules ou un principe actif marqués avec un radionucléide. Mais l'analyse peut également porter sur des cellules vivantes, et en temps réel, pour suivre différentes fonctions cellulaires telles que la division, la synthèse et la translocation de protéines, les flux de calcium intracellulaire, la synthèse d'ATP... Plusieurs aspects de la vie cellulaire sont étudiés au laboratoire grâce à ces biopuces. La prolifération cellulaire de cellules primaires ou de lignées est suivie par immunofluorescence dirigée contre la protéine PCNA (proliferating cell nuclear antigen) naturellement exprimée dans des cellules en prolifération, mais également contre un nucléotide modifié ajouté au milieu (5-bromo-2'-désoxy-uridine) incorporé dans l'ADN cellulaire lors de la phase $S$. La différenciation est suivie également par IF en choisissant d'autres marqueurs spécifiques du type cellulaire étudié comme, par exemple, la myosine dans des cellules myogéniques. L'apoptose, quant à elle, est suivie à l'aide d'un inhibiteur de caspases marqué par un fluorophore (Figure 3). À défaut de fournir des indications précises sur les mécanismes moléculaires impliqués dans les phénotypes étudiés, ces analyses devraient permettre d'accélérer significativement l'annotation fonctionnelle du génome.

Mais les applications des puces à cellules ne se limitent pas à l'exploration fonctionnelle. Elles sont, par exemple, adaptables à la mesure de l'affinité potentielle d'un ligand pour des protéines réceptrices exprimées à la surface des cellules transfectées. II suffit pour cela de déposer sur la puce les vecteurs d'expression de la collection de protéines étudiées et de suivre la fixation du ligand sur les îlots de cellules qui les expriment. La possibilité de réaliser des criblages à haut débit, tout en utilisant de très faibles volumes (et donc de faibles quantités de petites molécules à haute valeur ajoutée), rend ce format de biopuces particulièrement attractif pour l'industrie pharmaceutique.

Enfin, en ce qui concerne l'analyse en imagerie optique des puces à cellules, il convient de rappeler que ce format a été mis au point pour être adapté à tout type de microscope, pourvu que celui-ci soit doté des optiques 